\title{
CHERN-SIMONS INVARIANTS OF REDUCTIVE HOMOGENEOUS SPACES
}

\author{
BY \\ HAROLD DONNELLY
}

\begin{abstract}
The geometric characteristic classes of Chern-Simons are computed for certain connections on the canonical bundle and tangent bundle over a reductive homogeneous space. This includes globally symmetric spaces with the Levi-Civita connection of any bi-invariant metric.
\end{abstract}

Introduction. In recent papers [9], [11], [18], Chern and Simons have developed a theory of secondary characteristic classes on manifolds. These classes were calculated by Heitsch and Lawson on compact Lie groups with bi-invariant metric. One result of this paper is to extend these calculations to Riemannian symmetric spaces.

$\$ 1$ begins by quickly reviewing the main results of Chern and Simons. Later in this section we prove some general lemmas concerning extension of connections and Chern-Simons theory. In $\$ 2$ we state the properties of the transgression map. In $\$ 3$ some well-known results concerning invariant connections on reductive homogeneous spaces are recorded. $\$ 4$ gives the main result, Theorem 4.5, relating the Chern-Simons invariants of the bundle of bases over a reductive homogeneous space with the canonical affine connection of the second kind to those of those of the defining bundle with its canonical connection. $\$ 5$ specializes to symmetric spaces and Theorem 5.1 shows that the secondary classes are well-defined in some cases. In $\$ 6$ we treat Lie groups as symmetric spaces and in particular recover the main result of [12]. \$7 involves the calculation of secondary classes over Riemannian symmetric spaces fibered by the classical compact Lie groups. In $\$ 8$ we calculate the Chern-Simons invariants restricting conformal immersions for the spaces of $\$ 7$ and obtain the nontrivial invariants for $S U(2 k+1)$ and $S U(2 k+1) / S O(2 k+1)$.

The main idea of the calculation is that the Chern-Simons invariants for the bundle of bases over $G / H$ with canonical affine connection of the second kind are determined by the Chern-Simons invariants of the natural connection over the defining bundle. This last bundle may be treated using techniques from

Received by the editors April 7, 1975.

AMS (MOS) subject classifications (1970). Primary 53C35. 
algebraic topology and the usual theory of characteristic classes.

1. Chern-Simons theory. In recent papers S. S. Chern and J. Simons have studied secondary characteristic classes on manifolds. These classes are defined when the invariant polynomials $P(\Omega)$ of the Weil homomorphism vanish and the secondary classes have interesting geometrical properties.

In their joint work Chern and Simons associate to triples $(B, M, \phi)$ consisting of a principal bundle $B$ with connection $\phi$ over a manifold $M$ forms $\operatorname{TP}(\phi)$ on the bundle $B$ for each invariant polynomial $P \in I^{k}(G)$ of degree $k$ where $G$ is the group of the bundle. If we set $\Omega_{t}=t \Omega+\frac{1}{2}\left(t^{2}-t\right)[\phi, \phi]$ then by definition $T P(\phi)=k \int_{0}^{1} P\left(\phi, \Omega_{t}\right) d t$. A calculation shows $d T P(\phi)=P(\Omega)$. Thus if $P(\Omega)=0, T P(\phi)$ determines a real cohomology class in $B$. When $B$ is the bundle of bases over $M$ the forms $\operatorname{TP}(\phi)$ are invariant under conformal change of metric where $\phi$ is chosen as the Levi-Civita connection associated to a metric. The main result of [9] is the following:

THEOREM 1.1. Let $M^{n}$ be an n-dimensional Riemannian manifold. Let $d(M)$ $=\{E(M), M, \theta\}$ denote the $G l(n, R)$ basis bundle over $M$ equipped with the Riemannian connection $\theta$. A necessary condition that $M^{n}$ admit a conformal immersion in $R^{n+k}$ is that $P_{i}^{\perp}(\Omega)=0$ and $\left\{\frac{1}{2} T P_{i}^{\perp}(\theta)\right\} \in H^{4 i-1}(E(M), Z)$ for $i>[k / 2]$.

In a later paper [18], Simons defined characters associated to triples $(B, M, \phi)$. The idea was to define some geometric invariants in $M$ corresponding to the $T P(\phi)$ in $B$. Since the homology structure of $M$ is in general simpler than that of $B$ it is reasonable to expect that such invariants would be easier to apply.

For each pair $(P, u)$ where $P \in I^{1}(G)$ is an invariant polynomial of degree 1 and $u \in H^{21}\left(B_{G}, Z\right)$ such that $W(P)=r(u)$ with

$$
W: I^{1}(G) \rightarrow H^{21}\left(B_{G}, R\right)
$$

the Weil homomorphism and $r: H^{21}\left(B_{G}, Z\right) \rightarrow H^{21}\left(B_{G}, R\right)$ the coefficient homomorphism we have a character $S_{P, u}(\phi): Z_{2 l-1}(M) \rightarrow R / Z$. $S_{P, u}(\phi)$ have a natural definition on torsion cycles $x \subset M$. If $x \in Z_{2 l-1}^{\text {tor }}(M)$ and $\gamma$ $\in Z^{2 l-1}(M)$ represents the characteristic class $u$ let $y \in Z_{2 l}(M)$ be such that $\partial y=n x$. Then by definition $S_{P, u}(\phi)(x)=\overline{(P(\Omega) y-\gamma y) / n}$ where the bar denotes reduction mod $Z$. Simons proves by elementary arguments that this definition on torsion cycles is independent of the choices $y, \gamma$.

To define $S_{P, u}(\phi)$ for general cycles we must assume that $G$, the group of $B$, has a finite number of connected components. Then there is a classifying map of $(B, M, \phi)$ into a sufficient approximation of the classifying space $B_{G}$ with a canonical connection $\omega$. Now it is well known that $H^{\text {odd }}\left(B_{G}, R\right)=0$ since $G$ 
has a finite number of connected components. Then the image of any odd dimensional cycle in $M$ is a torsion cycle in $B_{G}$. Simons defines $S_{P, u}(\phi)$ by the given definition on torsion cycles and the requirement of naturality under connection preserving bundle maps. He proves that the definition is independent of the choice of classifying map.

The characters $S_{P, u}(\phi)$ determine the forms $P(\Omega)$ and the characteristic classes $u$. These characters determine $R / Z$ cohomology classes if and only if $P(\Omega)=0$.

The characters $S_{P, u}(\phi)$ are closely related to the forms $T P(\phi)$. In fact we have the following [18]:

TheOREM 1.2. If $\pi: B \rightarrow M$ is the projection then

$$
\pi^{*} S_{P, u}(\phi)=\overline{T P(\phi)} \mid Z_{2 l-1}(E)
$$

where the bar denotes reduction $\bmod Z$.

Corollary 1.3. If $S_{P, u}(\phi)=0$ then $\operatorname{TP}(\phi)$ is an integral class.

The analogue of Theorem 1.1 is given by Simons [18]:

THEOREM 1.4. Let $M$ be an n-dimensional Riemannian manifold. A necessary condition that it admit a conformal immersion in $R^{n+k}$ is that $S_{i}^{\perp}(\phi)=0$ for $i$ $>[k / 2]$.

For our later work we need some preliminary lemmas in Chern-Simons theory. They are established below.

LEMMA 1.5. Let $P$ be a principal $G_{1}$ bundle with connection $\phi$ and $f: G_{1} \rightarrow G_{2}$ a homomorphism. Define $P=P \times G_{2} /-$ the associated principal bundle with connection $\hat{\phi}$. The bar denotes the usual equivalence relation $(p, g)=\left(p h^{-1}\right.$, $f(h) g)$ and denote e the identity of $G_{2}$. Let $i_{f}: P \rightarrow \hat{P}$ be the map $p \rightarrow \overline{(p, e)}$. Then $i_{f}^{*} \hat{\phi}=f_{*} \phi$ where $f_{*}$ is the Lie algebra homomorphism induced by $f$.

Proof. For $p \in P$ and $Z \in \mathbb{S}_{1}\left(\right.$ resp. $\left.\mathbb{S}_{2}\right)$ denote by $\bar{Z}$ the vector field induced on the fiber containing $p$ (resp. $i p$ ).

(a) For $X \in \mathbb{B}_{1}$ if

$$
\begin{aligned}
\left(p e^{t X}\right) & =\overline{\left(p e^{t X}, e\right)}=\overline{\left(p, f\left(e^{t X}\right) e\right)}=\overline{\left(p, e f\left(e^{t X}\right)\right)}=\overline{(p, e)} f\left(e^{t X}\right) \\
& =\overline{(p, e) e^{\left(f_{*} X\right) t}}
\end{aligned}
$$

this shows $\left(i_{f}\right)_{*} \bar{X}=\overline{f_{*} X}$.

(b) The horizontal subspace at $i_{f} p$ is by definition the image of the horizontal space at $p$. Now let $\bar{X}$ be an arbitrary vertical vector $i_{f}^{*} \hat{\phi}(\bar{X})$ $=\hat{\phi}\left(\left(i_{f}\right)_{*} \bar{X}\right)=\hat{\phi}\left(\overline{f_{*} X}\right)=f_{*} X=f_{*} \phi(\bar{X})$. Let $Y$ be an arbitrary horizontal 
vector then

$$
\left(i_{f}^{*} \hat{\phi}\right)(Y)=\hat{\phi}\left(\left(i_{f}\right)_{*} Y\right)=0=f_{*} \phi(Y)
$$

Since any tangent vector to $P$ is the sum of a horizontal and a vertical vector this completes the proof.

COROLLARY 1.6. With the assumptions as in Lemma $1.5 i_{f}^{*} \hat{\Omega}=f_{*} \Omega$ where $\hat{\Omega}$ (resp. $\Omega$ ) is the curvature form of $\hat{\phi}$ (resp. $\phi)$.

Let $f: G_{1} \rightarrow G_{2}$ be as in Lemma 1.5. Then $f$ induces maps $f^{*}: I^{l}\left(G_{2}\right)$ $\rightarrow I^{l}\left(G_{1}\right), f^{*}: H^{q}\left(B_{G_{2}}\right) \rightarrow H^{q}\left(B_{G_{1}}\right)$.

LEMMA 1.7. For any $Q \in I^{l}(G)$ we have $\left(f^{*} Q\right)(\Omega)=Q(\hat{\Omega})$ considered as forms in the base manifold $M$.

Proof. $i_{f}^{*}(Q(\hat{\Omega}))=Q\left(i_{f}^{*} \hat{\Omega}\right)=Q\left(f_{*} \Omega\right)=\left(f^{*} Q\right)(\Omega)$. Now if $p, \hat{p}$ are the projections in $P, \hat{P}$ we have $\hat{p} i_{f}=p$. So for a form $\omega$ in the base $M$, $i_{f}^{*} \hat{p}^{*} \omega=p^{*} \omega$. The assertion follows.

We wish to relate the Simons characters of $P, \hat{P}$. For this we need the preliminary:

LEMMA 1.8. If $W(Q)=r(u)$ for $Q \in I^{l}\left(G_{2}\right), u \in H^{2 l}\left(B_{G_{2}}, Z\right)$ then if $f: G_{1}$ $\rightarrow G_{2}$ is a homomorphism $W\left(f^{*} Q\right)=r\left(f^{*} u\right)$.

Proof. Let $E_{G_{1}}$ be sufficiently $n$-classifying for $G_{1}$ and use $f$ to extend it to a bundle $\hat{E}$ with group $G_{2}$. If $\theta$ is the canonical connection on $E_{G}$ let $\hat{\theta}$ be the induced connection on $\hat{E}$. Let $\gamma: \hat{E} \rightarrow E_{G_{2}}$ be a connecting preserving for classifying map for $E$ where $E_{G_{2}}$ is $s$-classifying for $G_{2}$ with $s \geqslant n$. Then by definition $f^{*} u=\gamma^{*} u . \quad W\left(f^{*} Q\right)=\left(f^{*} Q\right)(\Omega)=i_{f}^{*} Q(\hat{\Omega})$ in the bundles $E$, $E$ where the third step in the proof follows from the proof of Lemma 1.7. Then in the space $B_{G_{1}}, W\left(f^{*} Q\right)=Q(\hat{\Omega})=r\left(\gamma^{*} u\right)=r\left(f^{*} u\right)$ where the third step follows by naturality of the connection preserving bundle map $\gamma$ and the relation $W(Q)=r(u)$.

LEMMA 1.9. With the notation as in Lemma 1.5

$$
S_{Q, u}(\hat{\phi})=S_{f^{*} Q, f^{*} u}(\phi)
$$

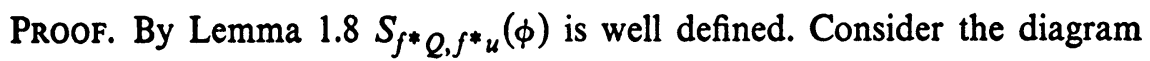
below:

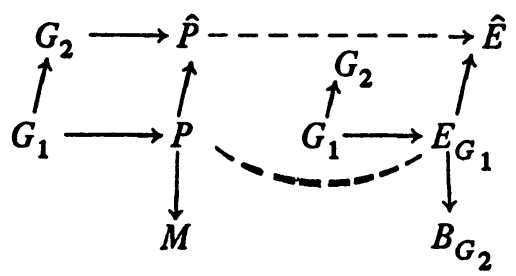


Notation is as in the proof of Lemma 1.8. The maps are obtained by choosing a classifying map $P \rightarrow E_{G_{1}}$ and extending it to a map $P \rightarrow E$. By naturality of the Simons character it is enough to verify the relation on the system $E_{G_{1}}, \hat{E}$. The real cohomology of $B_{G_{1}}$ vanishes in the relevant odd dimension. Thus all cycles are torsion cycles. Let $\partial y=n x$,

$$
\begin{aligned}
S_{f^{*} Q, f^{*} u}(\phi)(x) & =\overline{\left(f^{*} Q(\Omega)-f^{*} u\right)(y)} / n \\
& =\overline{(Q(\hat{\Omega})-u)(y) / n}=S_{Q, u}(\hat{\phi})(x)
\end{aligned}
$$

where the third step follows from $u(\hat{E})=f^{*} u\left(E_{G_{1}}\right)$ as in Lemma 1.8 by the definition of $f^{*}$.

LEMMA 1.10. With notation as in Lemma 1.5

$$
i_{f}^{*} T Q(\hat{\phi})=T\left(f^{*} Q\right)(\phi) .
$$

Proof. This follows easily from Lemma 1.5 , Corollary 1.6 , and the definition of $T P$.

2. The transgression. There is a well-known map $\tau$ called the transgression which will be important in this paper. Let $G$ be a compact Lie group and $\pi: E G \rightarrow B G$ its classifying object. For an arbitrary coefficient ring $\Lambda, \tau$ : $H^{i}(B G, \Lambda) \rightarrow H^{i-1}(G, \Lambda)$ with $i \geqslant 1 . \tau$ is defined as follows: Let $\gamma_{1}$ represent a class $\alpha_{1}$ in $H^{i}(B G, \Lambda) . \quad \pi^{*} \gamma_{1}$ is closed and therefore by acyclicity of $E G$ there exists $\gamma_{2}$ a cochain in $Z^{i-1}(E G, \Lambda)$ s.t. $\delta \gamma_{2}=\pi^{*} \gamma_{1}$. If $i: G \rightarrow E G$ is the inclusion of the fiber in the bundle then $\tau \alpha_{1}$ is the class $i^{*} \gamma_{2}$. It is straightforward to check that this definition of $\tau$ is independent of the choices made.

The following well-known lemma will be needed in the calculations made in the latter part of this paper:

LEMMA 2.1. $\tau$ maps products to zero.

PRoof. Let $\alpha=\alpha_{1} \alpha_{2} \in H^{i}(B G, \Lambda)$. Then if $\gamma_{1}$ represents $\alpha_{1}, \gamma_{2}$ represents $\alpha_{2}$, we have $\gamma_{1} \gamma_{2}$ representing $\alpha_{1} \alpha_{2}$. If $\delta \beta=\pi^{*} \gamma_{1}$ then $\delta\left(\beta \pi^{*} \gamma_{2}\right)=\left(\pi^{*} \gamma_{1}\right)$ $\cdot\left(\pi^{*} \gamma_{2}\right)=\pi^{*}\left(\gamma_{1} \gamma_{2}\right)$. But $i^{*}\left(\beta \pi^{*} \gamma_{2}\right)=\left(i^{*} \beta\right)\left(i^{*} \pi^{*} \gamma_{2}\right)=0$, since $i^{*} \pi^{*} \gamma_{2}$ $=0$.

If the coefficient ring is the real numbers then the transgression map is closely related to the forms $T P(\phi)$. In fact let $\pi: E \rightarrow B$ be a sufficiently close approximation to a classifying object. Then identifying the cohomology groups of $B$ in lower dimensions with those of $B_{G}$ we may define essentially the same transgression in $\pi: E \rightarrow B$. If $\phi$ is a connection on $\pi: E$ $\rightarrow B$ let $P(\Omega)$ for $\operatorname{deg} P$ sufficiently small be the Weil forms in the base. Then 
it is a direct consequence of the definitions that $\tau[P(\Omega)]=\left[i^{*} T P(\phi)\right]$. A straightforward calculation gives for $P \in I^{k}(G)$ :

$$
\tau_{p}=\left(-\frac{1}{2}\right)^{k-1} \frac{k !(k-1) !}{(2 k-1) !} P\left(\omega_{G},\left[\omega_{G}, \omega_{G}\right]\right)
$$

where $p=[P(\Omega)]$ and $\omega_{G}$ is the Maurer-Cartan form on $G$.

3. Connections on reductive homogeneous spaces. The material in this section is well known and more details may be found in [14] and [17].

A homogeneous space is called reductive if there is a decomposition

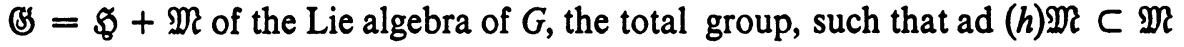
all $h \in H$, the fiber group. If $x H \in G / H$ then $x H \rightarrow g x H$ defines an action of $G$ on the homogeneous space $G / H$. Since for all $h \in H, h$ maps $e H$ to $e H$, the action of $h$ induces a representation called the isotropy representation of $H$ on the tangent space to $G / H$ at $e H$. If $\mathfrak{M}$ is identified with this tangent space then the isotropy representation is just the action of $H$ on $\mathfrak{M}$.

Let $p_{0}$ denote the identity coset $e H$. There exists a neighborhood $N^{*}$ of $p_{0}$ such that for $g \in G$ and $X \in \mathfrak{M}$ there is a vector field $X^{*}$ in $N^{*}$ defined by $\left(X^{*}\right)_{g p_{0}}=g_{*} X$ with $g$ an element of a local section and where on the right hand side $X$ is identified with an element of the tangent space at $p_{0}$. Then the fundamental existence theorem of [17] concerning invariant affine connections on $G / H$ is the following:

THEOREM 3.1. Let $G / H$ be a reductive homogeneous space with fixed decomposition of the Lie algebra $\mathbb{S}=\mathfrak{G}+\mathfrak{M}$, ad $(H) \mathfrak{M} \subset \mathfrak{M}$. There exists a one to one correspondence between the set of $G$ invariant affine connections on $G / H$ and the set of all bilinear functions $\alpha$ on $\mathfrak{M} \times \mathfrak{M}$ with values in $\mathfrak{D}$ which are invariant by $\operatorname{ad}(H)$, that is, ad $h(\alpha(X, Y))=\alpha(\operatorname{ad} h X, \operatorname{ad} h Y)$ for $X, Y \in \mathfrak{M}$ and $h$ $\in H$. The correspondence is given by

$$
\alpha(X, Y)=\left(\nabla_{Y^{*}} X^{*}\right)_{p_{0}} .
$$

We shall be interested in the connection given by $\alpha \equiv 0$. This is called the canonical affine connection of the second kind.

We shall also be concerned with a connection on the defining bundle $(G, G / H, H, \pi)$ over $G / H$. We define the canonical connection on this bundle by $\phi(X)=X_{\mathfrak{\Phi}}$ where $X$ is any left invariant vector field on $G$ and $X_{\mathfrak{W}}$ is its $\$$ component relative to the decomposition $\mathbb{S}=\mathfrak{G}+\mathfrak{R}$. This is easily seen to be a $G$ invariant connection where $G$ acts to the left.

4. Chern-Simons theory on reductive homogeneous spaces. In this section we establish our main result which relates the Simons character and forms $T P$ of the canonical affine connection of the second kind to those of the canonical 
connection on the defining bundle over $G / H$.

First we need some notation. Let $(B, G / H, G l(n), \pi, \omega)$ be the bundle of bases over $G / H$ with $\omega$ the canonical affine connection of the second kind and $\Omega$ the curvature form of $\omega$. Let $(G, G / H, H, p, \phi)$ be the defining bundle over $G / H$ with its canonical affine connection $\phi$ and $\Phi$ the curvature form of $\phi$. Denote $i_{s}: H \rightarrow G l(n)$ the isotropy representation. Choose left invariant vector fields $X_{i}, 1 \leqslant i \leqslant n$, on $G$ such that the $X_{i}$ span $\mathfrak{M}$.

We define a map $F: G \rightarrow B$ which is the principal tool used in the sequel.

$$
F(g)=\left(g H, P_{*}\left(X_{1}\right)_{g}, \ldots, P_{*}\left(X_{n}\right)_{g}\right)=\left(g H, g_{*}\left(X_{1}\right)_{e H}, \ldots, g_{*}\left(X_{n}\right)_{e H}\right) .
$$

LEMMA 4.1. $F(g h)=F(g) i_{s}(h)$.

Proof.

$$
\begin{aligned}
F(g h) & =\left(g H, g_{*} h_{*}\left(X_{1}\right)_{e H}, \ldots, g_{*} h_{*}\left(X_{n}\right)_{e H}\right) \\
& =\left(g H, g_{*}\left(X_{1}\right)_{e H}, \ldots, g_{*}\left(X_{n}\right)_{e H}\right) i_{s}(h)=F(g) i_{s}(h) .
\end{aligned}
$$

LEMMA 4.2. Define a connection $\gamma$ on $B$ by choosing the horizontal subspace at $F(g)$ to be the image $F_{*}\left(\mathfrak{M}_{g}\right)$ and extending by right invariance under $G l(n)$. Then $\gamma$ is a well-defined $G$ invariant connection on $B$.

Proof. $\pi F$ is onto $G / H$ so we have a subspace $F_{*}\left(\mathfrak{M}_{g}\right)$ at a point on each fiber. If $\pi F\left(g_{1}\right)=\pi F\left(g_{2}\right)$ then $g_{1}=g_{2} h$ for some $h \in H$. Now by Lemma 4.1, $F(g h)=F(g) i_{s}(h)$ and $F_{*}\left(\mathfrak{M}_{g h}\right)=F_{*}\left(\mathfrak{M}_{g}\right)\left(i_{s}(h)\right)_{*}$. So our definition can be extended to be right invariant on the fiber.

To show that we have defined a connection we need only verify that the subspace we have defined is complementary at each point to the tangent space of the fiber. Fix $g \in G$. Then $F_{*}\left(\mathfrak{M}_{g}\right)$ has dimension at most $n$ and since $\pi_{*} F_{*}\left(\mathfrak{M}_{g}\right)=g_{*} P_{*}\left(\mathfrak{M}_{e}\right)=g_{*}\left(T_{e H}\right)$ it is exactly of dimension $n$ and is complementary to the tangent space of the fiber.

To check the invariance under the left action of $G$ on $G / H$ note that

$$
\begin{aligned}
F\left(g_{1} g_{2}\right) & =\left(g_{1} g_{2} H, g_{1^{*}} g_{2^{*}}\left(X_{1}\right)_{e H}, \ldots, g_{1^{*}} g_{2^{*}}\left(X_{n}\right)_{e H}\right) \\
& =g_{1^{*}}\left(g_{2} H, g_{2^{*}}\left(X_{1}\right)_{e H}, \ldots, g_{2^{*}}\left(X_{n}\right)_{e H}\right)=g_{1^{*}} F_{*}\left(\mathbb{R}_{g_{2}}\right) .
\end{aligned}
$$

Then $F_{*}\left(\mathfrak{M}_{g_{1} g_{2}}\right)=F_{*}\left(g_{1^{*}} \mathfrak{M}_{g_{2}}\right)=g_{1^{*}} F_{*}\left(\mathfrak{M}_{g_{2}}\right)$ giving the required result.

REMARK. Note that by definition $\mathfrak{M}_{g}$ is the horizontal subspace at $g$ for the canonical connection $\phi$.

LEMMA 4.3. The connection $\gamma$ defined in Lemma 4.2 is the canonical affine connection of the second kind $\omega$ on $B$. 
Proof. Since both $\gamma$ and $\omega$ are $G$-invariant it suffices to work in a neighborhood $N^{*}$ as described in $\S 3$. Let $\nabla$ denote covariant differentiation with respect to $\gamma$ and let $\sigma$ be the local section of $B$ given by $X_{1}^{*}, \ldots, X_{n}^{*}$ with notation as in $\$ 3$.

Since by definition of $\gamma,\left.\sigma_{*} X_{1}^{*}\right|_{p_{0}}$ are horizontal. By Theorem 3.1, $\gamma$ is the canonical affine connection of the second kind $\omega$.

LEMMA 4.4. Let $\hat{G}$ be the principal $G l(n)$ bundle which is associated to $G$ by the isotropy representation $i_{s}$. Then $\hat{G}=G \times G l(n) /-$. Denote by $j: G \rightarrow \hat{G}$ the natural map $j(g, e)=(\overline{g, e})$.

Now define a map $F: \hat{G} \rightarrow B$ by the conditions

(i) $\hat{F}_{j}(g, e)=F(g)$ and

(ii) $\hat{F}(\hat{g} \omega)=(\hat{F}(\hat{g})) \omega$

for all $\omega \in G l(n)$. Then $F$ is a connection preserving bundle map.

Proof. First we must check that $\hat{F}$ is well defined. That is, if $j(\bar{g}, e)$ $=j(g, e) \omega$ then $F(\bar{g})=F(g) \omega$. Now $j(\bar{g}, e), j(g, e)$ lie in the same fiber if and only if $\bar{g}, g$ lie in the same fiber. Then $\bar{g}=g h$ for some $h \in H$. $j(\bar{g}, e)$ $=j(g h, e)=j\left(g, i_{s}(h) e\right)=j(g, e) i_{s}(h)$. Also $F(\bar{g})=F(g h)=F(g) i_{s}(h)$ by Lemma 4.1. Therefore $\hat{F}$ is well defined.

To show that $\hat{F}$ is connection preserving note that since $\hat{F}$ commutes with the action of $G l(n)$ it is enough to check that the image of the horizontal space at each $j(g, e)$ is the horizontal space at $F(g)$. Now the horizontal space $H$ at $j(g, e)$ is by definition $j_{*}\left(\mathfrak{M}_{g}\right)$. So $\hat{F}_{*}(H(j(g, e)))=\hat{F}_{*} j_{*} \mathfrak{M}_{g}=F_{*} \mathfrak{M}_{g}$. The result then follows from Lemmas 4.2 and 4.3.

THEOREM 4.5. Let $Q \in I^{l}(G l(n)), u \in H^{2 l}\left(B_{G l(n)}, Z\right)$ such that $W(Q)$ $=r(u)$, then

(i) $Q(\Omega)=\left(i_{s}^{*} Q\right)(\Phi)$

(ii) $F^{*} T Q(\omega)=T\left(i_{s}^{*} Q\right)(\phi)$,

(iii) $S_{Q, u}(\omega)=S_{i_{s}^{*} Q, i_{s}^{*} u}(\phi)$.

Proof. (i) follows from Lemmas 1.7 and 4.4 since $F$ of Lemma 4.4 induces the identity map of the base spaces.

(ii) $F^{*} T Q(\omega)=j^{*} \hat{F}^{*} T Q(\omega)$ with notation as in Lemma 4.4. The result now follows from Lemmas 1.10 and 4.4 applying naturality of $T P$ under connection preserving bundle maps.

(iii) follows from Lemmas $1.9,4.4$, the fact that $\hat{F}$ induces the identity map on base spaces and naturality of the Simons character under connection preserving bundle maps.

5. Symmetric spaces. The symmetric spaces with their canonical Lie algebra decomposition are all reductive homogeneous spaces. Further on a symmetric space $G / H$ the canonical affine connection of the second kind coincides with 
the canonical affine connection of the first kind [17] and by Theorem 15.6 of [17] this is the connection induced by any $G$-invariant metric. Such metrics always exist if $H$ is compact.

We proceed to do some computations on the defining bundle $(G, G / H, H, p$, $\phi)$ over a symmetric space $G / H$. Let $P \in I^{d}(H)$ be any $H$ invariant polynomial.

$$
\mathbb{S}=\mathfrak{S}+\mathfrak{M} \text { with }[\mathfrak{\&}, \mathfrak{\&}] \subset \mathfrak{\&},[\mathfrak{\&}, \mathfrak{M}] \subset \mathfrak{M},[\mathfrak{M}, \mathfrak{M}] \subset \mathfrak{\&} .
$$

Let $X, Y$ be left invariant vector fields on $G$.

$$
\begin{aligned}
& \phi(X)=X_{\S}, \\
& d \phi(X, Y)=-\phi(X, Y)=-[X, Y]_{\mathfrak{Q}}=-\left[X_{\mathfrak{R}}, Y_{\mathfrak{W}}\right]-\left[X_{\mathfrak{\$}}, Y_{\$}\right], \\
& \frac{1}{2}[\phi, \phi](X, Y)=\left[X_{\mathfrak{\$}}, Y_{\mathfrak{\$}}\right] \text {, } \\
& \Phi(X, Y)=\left(d \phi+\frac{1}{2}[\phi, \phi]\right)(X, Y)=-\left[X_{\mathfrak{M}}, Y_{\mathfrak{M}}\right], \\
& \Phi_{t}(X, Y)=\left(t \Phi+\frac{1}{2}\left(t^{2}-t\right)[\phi, \phi]\right)(X, Y) \\
& =-t\left[X_{\mathfrak{M}}, Y_{\mathfrak{M}}\right]+\left(t^{2}-t\right)\left[X_{\mathfrak{\$}}, Y_{\mathfrak{\$}}\right] .
\end{aligned}
$$

Define forms $\gamma_{1}: \& \times \& \rightarrow \varsubsetneqq$ by $\gamma_{1}(X, Y)=\left[X_{\mathfrak{M}}, Y_{\mathfrak{M}}\right], \gamma_{2}: \& \times \& \rightarrow \$$ by $\gamma_{2}(X, Y)=\left[X_{\S}, Y_{\S}\right]$. Then

$$
\begin{aligned}
& T P(\phi)=d \int_{0}^{1} P\left(\phi, \Phi_{t}\right) d t \\
& =d \int_{0}^{1} \sum_{r=0}^{d-1}(-1)^{r} t^{r}\left(t^{2}-t\right)^{d-r-1}\left(\begin{array}{c}
d-1 \\
r
\end{array}\right) P(\phi, \overbrace{\gamma_{1}, \ldots, \gamma_{1}}^{r} \gamma_{2}) d t \\
& =\sum_{r=0}^{d-1} P(\phi, \overbrace{\gamma_{1}, \ldots, \gamma_{1}}^{r}, \gamma_{2}) \int_{0}^{1} d(-1)^{r} t^{r}\left(t^{2}-t\right)^{d-r-1}\left(\begin{array}{c}
d-1 \\
r
\end{array}\right) d t \text {. }
\end{aligned}
$$

Set

$$
\begin{aligned}
W_{d, r} & =d\left(\begin{array}{c}
d-1 \\
r
\end{array}\right)(-1)^{r} \int_{0}^{1} t^{d-1}(1-t)^{d-r-1} d t(-1)^{d-r-1} \\
& =d\left(\begin{array}{c}
d-1 \\
r
\end{array}\right)(-1)^{d-1} \frac{(d-1) !(d-r-1) !}{(2 d-r-1) !} \\
& =(-1)^{d-1} \frac{d !(d-1) !}{r !(2 d-r-1) !} .
\end{aligned}
$$

Then

$$
T P(\phi)=\sum_{r=0}^{d-1} W_{d, r} P(\phi, \overbrace{\gamma_{1}, \ldots, \gamma_{1}}^{r}, \gamma_{2}) .
$$

We have the following result which shows that in some cases the secondary invariants of Chern-Simons define cohomology classes on symmetric spaces.

THEOREM 5.1. Suppose $P \in I(H)$ is the restriction of an invariant polynomial $P \in I(G)$ then $P(\Phi)=0$. 
Proof. Let $\omega_{\mathfrak{M}}$ : \&S $\rightarrow$ \&S be the \&S-valued one form defined by projection of a left-invariant vector field on $G$ along $\mathfrak{S}$. Then

$$
\begin{aligned}
P(\Phi)= & (-1)^{d} P\left(\left[\omega_{\mathfrak{M}}, \omega_{\mathfrak{M}}\right]\right)=\operatorname{Ad}_{\omega_{\mathfrak{R}}}(-1)^{d} P\left(\omega_{\mathfrak{M}},\left[\omega_{\mathfrak{M}}, \omega_{\mathfrak{M}}\right]\right) \\
& +(d-1)(-1)^{d} P\left(\omega_{\mathfrak{M}},\left[\omega_{\mathfrak{M}},\left[\omega_{\mathfrak{M}}, \omega_{\mathfrak{R}}\right]\right],\left[\omega_{\mathfrak{M}}, \omega_{\mathfrak{R}}\right]\right)=0
\end{aligned}
$$

where the first term vanishes by $\operatorname{Ad} G$ invariance and the second term vanishes by the Jacobi identity $[\theta,[\theta, \theta]]=0$ for any (S)-valued one form $\theta$.

For general $P$ the author has been unable to determine the $\operatorname{TP}(\phi)$ except on a case by case basis $(\$ \S 6,7)$. However if the degree of $P$ is low we have

Proposition 5.2. Suppose the symmetry of the symmetric Lie algebra is induced by an inner automorphism of $G$. Then

(i) If $P \in I^{1}(H)$ is the restriction of an $I(G)$ invariant polynomial then $T P(\phi)=\tau p$ where $p \in H^{2}\left(B_{G}, R\right)$ is the element corresponding to $P$.

(ii) If $P \in I^{2}(H)$ is the restriction of an $I^{2}(G)$ invariant polynomial $P$ then $T P(\phi)=\tau p$ where $p \in H^{4}\left(B_{G}, R\right)$ is the element corresponding to $P$.

PROOF. $\omega_{\mathfrak{W}}=\phi$ the projection on $H$ along $\mathfrak{M} ; \omega_{G}$ the Maurer-Cartan form of $G$.

$$
\begin{aligned}
T P(\phi) & =P(\phi)=P\left(\omega_{\mathfrak{S}}\right)=P\left(\omega_{G}-\omega_{\mathfrak{R}}\right) \\
& =P\left(\omega_{G}\right)-P\left(\omega_{\mathfrak{M}}\right)=P\left(\omega_{G}\right)=\tau p
\end{aligned}
$$

where $P\left(\omega_{\mathfrak{M}}\right)=P\left(-\omega_{\mathfrak{R}}\right)$ by applying the symmetry and by the formula of (2).

(ii)

$$
\begin{aligned}
& T P(\phi)=W_{2,0} P\left(\phi, \gamma_{2}\right)+W_{2,1} P\left(\phi, \gamma_{1}\right) \\
& =\frac{1}{3} P\left(\omega_{\mathfrak{Q}},\left[\omega_{\mathfrak{W}}, \omega_{\mathfrak{Q}}\right]\right)-P\left(\omega_{\mathfrak{W}}\left[\omega_{\mathfrak{R}}, \omega_{\mathfrak{R}}\right]\right), \\
& \tau p=-\frac{1}{3} P\left(\omega_{G},\left[\omega_{G}, \omega_{G}\right]\right) \\
& =-\frac{1}{3} P\left(\omega_{\mathfrak{Q}}+\omega_{\mathfrak{R}},\left[\omega_{\mathfrak{W}}, \omega_{\mathfrak{q}}\right]+\left[\omega_{\mathfrak{N}}, \omega_{\mathfrak{M}}\right]+2\left[\omega_{\mathfrak{Q}}, \omega_{\mathfrak{M}}\right]\right) \\
& =-\frac{1}{3} P\left(\omega_{\mathfrak{q}},\left[\omega_{\mathfrak{q}}, \omega_{\mathfrak{q}}\right]\right)-\frac{1}{3} P\left(\omega_{\mathfrak{q}},\left[\omega_{\mathfrak{q}}, \omega_{\mathfrak{R}}\right]\right) \\
& -\frac{2}{3} P\left(\omega_{\mathfrak{q}},\left[\omega_{\mathfrak{R}}, \omega_{\mathfrak{M}}\right]\right)-\frac{1}{3} P\left(\omega_{\mathfrak{R}},\left[\omega_{\mathfrak{q}}, \omega_{\mathfrak{\wp}}\right]\right) \\
& -\frac{1}{3} P\left(\omega_{\mathfrak{M}},\left[\omega_{\mathfrak{M}}, \omega_{\mathfrak{R}}\right]\right)-\frac{2}{3} P\left(\omega_{\mathfrak{R}},\left[\omega_{\mathfrak{S}}, \omega_{\mathfrak{M}}\right]\right) \\
& =-\frac{1}{3} P\left(\omega_{\mathfrak{W}},\left[\omega_{\mathfrak{\$}}, \omega_{\mathfrak{Q}}\right]\right)-P\left(\omega_{\mathfrak{W}},\left[\omega_{\mathfrak{W}}, \omega_{\mathfrak{R}}\right]\right) \text {. }
\end{aligned}
$$


So $T P(\phi)=\tau p$. The last step uses Ad $G$ invariance in setting $P\left(\omega_{\mathfrak{N}},\left[\omega_{\mathfrak{S}}, \omega_{\mathfrak{R}}\right]\right)$ $=P\left(\omega_{\mathfrak{Q}},\left[\omega_{\mathfrak{Q}}, \omega_{\mathfrak{M}}\right]\right)$.

6. Lie groups. In this section we consider the defining bundle ( $G \times G, G, \Delta G$, $p, \phi)$ exhibiting a Lie group as a symmetric space $(\Delta G$ denotes the diagonal in $G \times G)$. If $s: G \rightarrow G \times G$ by $(\overline{g, e}) \rightarrow(g, e)$ is the canonical section of this bundle we compute the forms $s^{*} T P(\phi)$. This permits we us recover the main result of [12] from a somewhat different viewpoint.

Let $(B, G l(n), G, \pi, \omega)$ be the bundle of bases over $G$ with $\omega$ the connection associated to a $G$ bi-invariant metric. To say a metric is bi-invariant under the action of $G$ is the same as saying it is $G \times G$ invariant when considered as a metric on a symmetric space.

THEOREM 6.1. For any $Q \in I(G)$ we have $Q(\Phi)=0$.

Proof. Let \&s $\oplus$ \&S be the Lie algebra of $G \times G$. Define $\bar{Q} \in I(G \times G)$ by $\bar{Q}\left(x_{1} \oplus x_{2}\right)=\frac{1}{2}\left(Q\left(x_{1}\right)+Q\left(x_{2}\right)\right)$. Then $\bar{Q}$ restricts to $Q$ on $G$. The result then follows from Theorem 5.1.

Corollary 6.2. For any $Q \in I(G l(n))$ we have $Q(\Omega)=0$.

PRoof. Immediate from Theorem 6.1 and Theorem 4.5(i).

We now do some calculations in the defining bundle. Let us denote the section $(\overline{g, e}) \rightarrow(g, e)$ by $s$.

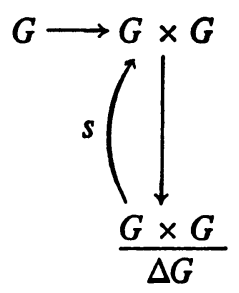

Let $\left(X_{1}, X_{2}\right) \in \mathbb{B} \oplus \oplus$ \&, the Lie algebra of $G \times G$.

$$
\left(X_{1}, X_{2}\right)=\frac{1}{2}\left(\left(X_{1}+X_{2}, X_{1}+X_{2}\right)+\left(X_{1}-X_{2}, X_{2}-X_{1}\right)\right)
$$

exhibits a decomposition of $\left(X_{1}, X_{2}\right)$ into $\mathfrak{G}$ and $\mathfrak{M}$ components.

$$
\left[\left(X_{1}, X_{2}\right),\left(Y_{1}, Y_{2}\right)\right]=\left(\left[X_{1}, Y_{1}\right],\left[X_{2}, Y_{2}\right]\right) .
$$

Let $\omega_{G}$ denote the Maurer-Cartan form on $G$.

$$
\begin{aligned}
\left(s^{*} \phi\right)(X) & =\frac{1}{2} X=\frac{1}{2} \omega_{G}(X), \\
\left(s^{*} \Phi\right)(X, Y) & =-\left[\frac{1}{2} X, \frac{1}{2} Y\right]=-\frac{1}{8}\left[\omega_{G}, \omega_{G}\right](X, Y), \\
\left(s^{*} \gamma_{1}\right)(X, Y) & =\frac{1}{8}\left[\omega_{G}, \omega_{G}\right](X, Y), \\
\left(s^{*} \gamma_{2}\right)(X, Y) & =\frac{1}{8}\left[\omega_{G}, \omega_{G}\right](X, Y) .
\end{aligned}
$$


THEOREM 6.3. For any $Q \in I^{k}(G)$ we have

$$
s^{*} T Q(\phi)=\frac{1}{2} \tau q,
$$

$q \in H^{2 k}(B G, R)$ is the class associated to $Q$. If $Q$ is an integral polynomial, that is, $q$ is in the image of the coefficient homomorphism

$$
r: H^{2 k}(B G, Z) \rightarrow H^{2 k}(B G, R),
$$

then $S_{Q, q}(\phi) \overline{\frac{1}{2} \tau q}$ where the bar denotes the coefficient homomorphism $H^{2 k-1}(G, R) \rightarrow H^{2 k-1}(G, R / Z)$.

Proor.

$$
\begin{aligned}
s^{*} T Q(\phi) & =s^{*} \sum_{r=0}^{k-1} W_{k, r} Q(\phi, \overbrace{\gamma_{1}, \ldots, \gamma_{1}, \gamma_{2}}^{r}) \\
& =\sum_{r=0}^{k-1} W_{k, \frac{1}{2}}\left(\frac{1}{8}\right)^{k-1} Q\left(\omega_{G},\left[\omega_{G}, \omega_{G}\right]\right) \\
& =\frac{1}{2}\left(-\frac{1}{2}\right)^{k-1} \frac{k !(k-1) !}{(2 k-1) !} Q\left(\omega_{G},\left[\omega_{G}, \omega_{G}\right]\right)=\frac{1}{2} \tau q .
\end{aligned}
$$

This shows the first part.

$$
S_{Q, q}(\phi)=1^{*} S_{Q, q}(\phi)=s^{*} p^{*} S_{Q, q}(\phi)=s^{*} \overline{T Q(\phi)}=\overline{s^{*} T Q(\phi)}=\overline{\frac{1}{2} \tau q,}
$$

where the third step uses Theorem 1.2.

CoRollary 6.4. For any $Q \in I^{k}(G l(n))$ and any section $\sigma$ of the bundle of bases of $G$ by left-invariant vector fields $\sigma^{*}(T Q(\omega))=\operatorname{Ad}^{*}\left(\frac{1}{2} \tau q\right)$ where Ad: $G$ $\rightarrow G l(n)$ is the adjoint representation. If $Q$ is integral then $S_{Q, q}(\omega)=0$.

Proof. If $F: G \times G \rightarrow B$ as in (4) then $\sigma=F s$ is a section of $B$. Further by proper choice of the vector fields $X_{1}, \ldots, X_{n}$ as in the beginning of (4) any section $\sigma$ by left invariant vector fields is given by some such $F$. $\sigma^{*}(T Q(\omega))$ $=S^{*} F^{*}(T Q(\omega))=S^{*}\left(T\left(i_{s}^{*} Q\right)(\phi)\right)$ where the last step follows from Theorem 4.5 (ii). Now $i_{s}$ may be identified with Ad in this case.

$$
\sigma^{*}(T Q(\omega))=s^{*}\left(T\left(\operatorname{Ad}^{*} Q\right)(\phi)\right)=\frac{1}{2} \tau\left(\operatorname{Ad}^{*} q\right)=\operatorname{Ad}^{*}\left(\frac{1}{2} \tau q\right)
$$

where the last step follows by naturality of $\tau$.

For the second part by Theorem 4.5 (iii) $S_{Q q}(\omega)=S_{\mathrm{Ad}^{*} Q, \mathrm{Ad}^{*} q}(\phi)$ $=\frac{1}{2} \tau\left(\operatorname{Ad}^{*} q\right)$ using Theorem 6.3. So $S_{Q, q}(\omega)=\overline{\operatorname{Ad}^{*}\left(\frac{1}{2} \tau q\right) \text {. But for } G l(n) \frac{1}{2} \tau q \text { is }}$ always integral [6] so $S_{Q, q}(\omega)=0$ by the long exact sequence of coefficient homomorphisms corresponding to $0 \rightarrow Z \rightarrow R \rightarrow R / Z \rightarrow 0$.

Corollary 6.4 is the main result of [12]. 
7. Secondary classes on symmetric spaces. In this section we compute some secondary classes in the defining bundle over the compact irreducible Riemannian symmetric spaces fibered by the classical groups other than the groups themselves which are treated in [12] and in \$6. Specifically we consider canonical generating polynomials $Q \in I(G)$. Then by Theorem $5.1, T\left(i^{*} Q\right)$ - $(\phi)$ where $i: H \rightarrow G$ is the inclusion always defines a cohomology class in $G$ corresponding to the defining bundle over $G / H$. The following proposition shows that it is enough to treat such a generating set.

Proposition 7.1. If $S=R Q$ is the product of G-invariant polynomials $R$ and $Q$ then $T\left(i^{*} S\right)(\phi)$ is the zero class.

Proof. By $[11],\left[T\left(i^{*} S\right)(\phi)\right]=\left[T\left(i^{*} R\right)(\phi) Q(\Phi)\right]=0$ since $Q(\Phi)=0$ by Theorem 5.1.

The list of spaces considered in this section is: $F_{n}=S O(2 n) / U(n), n>2$ : $G_{n}=\operatorname{Sp}(n) / U(n), n>2$; the Grassmannian $U(p+q) / U(p) \times U(q)$; the Grassmannian $\mathrm{Sp}(p+q) / \mathrm{Sp}(p) \times \mathrm{Sp}(q), p+q>2$; the Grassmannian $S O(p+q) / S O(p) \times S O(q), p+q>4$, treated in three cases depending upon the parity of $p$ and $q ; S U(n) / S O(n)$ treated in three cases depending upon the parity of $n ; S U(2 n) / \mathrm{Sp}(n), n>1$.

Before we begin the computations some preliminary remarks about notation and the devices used are required.

If $T \subset G$ is a maximal torus of a compact Lie group then the map $j^{*}: H^{*}\left(B_{G}, R\right) \rightarrow H^{*}\left(B_{T}, R\right)$ is known to be injective [4]. In the following calculation we will identify elements of $H^{*}\left(B_{G}, R\right)$ with their images in $H^{*}\left(B_{T}, R\right)$.

If $x_{1}, \ldots, x_{n}$ are variables and $\sigma_{j}\left(x_{1}, \ldots, x_{n}\right)$ denotes the $j$ th elementary symmetric function in the $x_{i}$ and $S_{j}$ the sum of $j$ th powers then [19]:

$$
s_{l}-S_{l-1} \sigma_{1}+S_{l-2} \sigma_{2}+\cdots+(-1)^{l-1} S_{1} \sigma_{l-1}+(-1)^{l} l \sigma_{l}=0 .
$$

This fact will often be used in conjunction with Lemma 2.1 in the sequel.

For notation we have $i: H \rightarrow G$ the inclusion and one of the maps $H^{*}\left(B_{G}\right) \rightarrow H^{*}\left(B_{H}\right), H^{*}(G) \rightarrow H^{*}(H), I(G) \rightarrow I(H)$ depending on the context. For $Q \in I(G), T Q(\phi)$ means $T\left(i^{*} Q\right)(\phi)$. All cohomology groups are understood to be with real coefficients.

(a) $F_{n}=S O(2 n) / U(n), n>2$. Then if $P_{i}$ and $\chi$ are the Pontryagin forms and Euler form we have $T P_{i}(\phi)=\tau p_{i}$ for $i \leqslant[n / 2]$ and $T \chi(\theta)=\tau \chi$. Let $T \subset U(n) \subset S O(2 n)$ where $T$ is the standard maximal torus of $S O(2 n)$. Now the generators of $H^{*}(S O(n))$ are the transgressions of the generators of $H^{*}\left(B_{S O(n)}\right)$ and these may be identified with $p_{j}=\sigma_{j}\left(\theta_{1}^{2}, \ldots, \theta_{n}^{2}\right), \chi=\theta_{1}, \ldots$, $\theta_{n}$ for $j=1, \ldots, n-1$ where $\theta_{i}$ are the generators of $H^{*}\left(B_{T}\right)$. The generators of $H^{*}(U(n))$ are the transgressions of the generators of $H^{*}\left(B_{U(n)}\right)$ and 
these may be identified with $c_{j}=\sigma_{j}\left(\theta_{1}, \ldots, \theta_{n}\right), j=1, \ldots, n$, where $\theta_{i}$ are again the generators of $H^{*}\left(B_{T}\right)$. Consider the following diagram:

$$
\begin{gathered}
H^{*}(U(n)) \stackrel{i^{*}}{\longleftarrow} H^{*}(S O(2 n)) \\
H^{*}\left(B_{U(n)}\right) \stackrel{i^{*}}{\longleftarrow} H^{*}\left(B_{S O(2 n)}\right) \\
i^{*} \tau \chi=\tau i^{*} \chi=\tau c_{n}, \\
i^{*} \tau p_{j}=\tau i p_{j}=\tau \sigma_{j}\left(\theta_{1}^{2}, \ldots, \theta_{n}^{2}\right) \\
=\tau(-1)^{j+1 / j} S_{j}\left(\theta_{1}^{2}, \ldots, \theta_{n}^{2}\right) \\
=\tau(-1)^{j+1 / j}(-1) 2 j \sigma_{2 j}\left(\theta_{1}, \ldots, \theta_{n}\right)=2(-1)^{j} \tau c_{2 j} .
\end{gathered}
$$

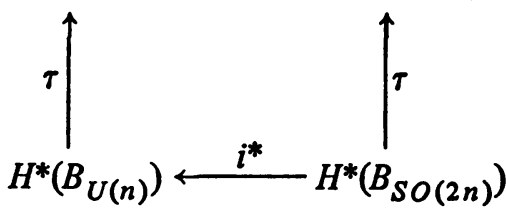

This shows that in dimensions less than or equal to $2 n-1$ restriction to the fiber is an injection on the cohomology level. Now $i^{*} T \chi(\theta)=\tau i^{*} \chi=\tau c_{n}$ $\cdot i^{*} T P_{j}(\theta)=\tau i^{*} p_{j}=2(-1)^{j} \tau c_{2 j}$. Therefore $T \chi(\theta)=\tau \chi, T P_{j}(\theta)=\tau p_{j}$ for $j$ $\leqslant[n / 2]$.

(b) $G_{n}=\mathrm{Sp}(n) / U(n), n>2$. Then if $P_{i}$ is the canonical symplectic Pontryagin form we have $T P_{i}(\theta)=\tau p_{i}$ for $i \leqslant[n / 2]$. Now $T \subset U(n) \subset \mathrm{Sp}(n)$ the generators of $H^{*}(U(n))$ are as described in $\$ 2$. The generators of $H^{*}(U(n))$ are the transgressions of the generators of $H^{*}\left(B_{\mathrm{Sp}(n)}\right)$ which may be identified with $p_{j}=\sigma_{j}\left(\theta_{1}^{2}, \ldots, \theta_{n}^{2}\right), j=1, \ldots, n$, where $\theta_{i}$ are the generators of $H^{*}\left(B_{T}\right)$. Consider the commutative diagram:

$$
\begin{gathered}
H^{*}(U(n)) \stackrel{i^{*}}{\longleftarrow} H^{*}(\operatorname{Sp}(n)) \\
\prod^{*}\left(B_{U(n)}\right) \stackrel{i^{*}}{\longleftarrow} H^{*}\left(B_{\mathrm{Sp}(n)}\right) \\
i^{*} \tau p_{j}=\tau i^{*} p_{j}=\tau s_{j}\left(\theta_{1}^{2}, \ldots, \theta_{n}^{2}\right)=\tau(-1)^{j+1} / j s_{j}\left(\theta_{1}^{2}, \ldots, \theta_{n}^{2}\right) \\
=\tau(-1)^{j+1} / j(-1)(2 j) \sigma_{2 j}\left(\theta_{1}, \ldots, \theta_{n}\right)=(-1)^{j} 2 \tau c_{2 j} .
\end{gathered}
$$

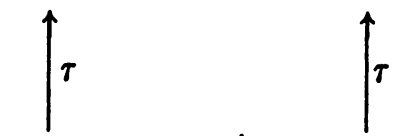

This shows that in dimensions less than or equal to $2 n-1$ restriction to the fiber is an injection on the cohomology level. Now $i^{*} T P_{i}(\phi)=\tau i^{*} p_{i}$ $=(-1)^{i} \tau c_{2 i}$. Therefore $T P_{i}(\phi)=\tau p_{i}$ for $i \leqslant\left[N_{2}\right]$. 
(c) The Grassmannian $U(p+q) / U(p) \times U(q)$. Then if $C_{r}$ are the Chern forms on $U(p+q)$ we have $T C_{r}(\phi)=\tau c_{p}$ for $r \in \max (p, q)$. Let $T \subset U(p)$ $\times U(q) \subset U(p+q)$ be the standard maximal torus. Then the generators of $H^{*}(U(p+q))$ are the trangressions of the $C_{r}$ which may be identified with $\sigma_{r}\left(\theta_{1}, \ldots, \theta_{p+q}\right), r=1, \ldots, p+q$. The generators of $H^{*}(U(p) \times U(q))$ are the transgressions of the $c_{j}^{\prime} \times 1,1 \times c_{k}^{\prime \prime}$ which may be identified with

$$
\sigma_{j}\left(\theta_{1}, \ldots, \theta_{p}\right) \times 1, \quad 1 \times \sigma_{k}\left(\theta_{p+1}, \ldots, \theta_{p+q}\right)
$$

for $j=1, \ldots, p$ and $k=1, \ldots, q$. Consider the commutative diagram:

$$
\begin{gathered}
H^{*}(U(p) \times U(q)) \stackrel{i^{*}}{\prod_{i} H^{*}(U(p+q))} \\
H^{*}\left(B_{U(p) \times U(q)}\right) \stackrel{i^{*}}{\longleftarrow} H^{*}\left(B_{U(p+q)}\right) \\
i^{*} \tau c_{r}=i^{*} \tau \sigma_{r}\left(\theta_{1}, \ldots, \theta_{p+q}\right)=i^{*} \frac{(-1)^{r+1}}{r} \tau S_{r}\left(\theta_{1}, \ldots, \theta_{p+q}\right) \\
=\frac{(-1)^{r+1}}{r}\left(\tau S_{r}\left(\theta_{1}, \ldots, \theta_{p}\right) \otimes 1+1 \otimes \tau S_{r}\left(\theta_{p+1}, \ldots, \theta_{p+q}\right)\right) \\
=\frac{(-1)^{r+1}}{r} \frac{r}{(-1)^{r+1}}\left(\tau \sigma_{r}\left(\theta_{1}, \ldots, \theta_{p}\right) \otimes 1+1 \times \sigma_{r}\left(\theta_{p+1}, \ldots, \theta_{p+q}\right)\right) \\
=\tau c_{r}^{\prime} \otimes 1+1 \otimes \tau c_{r}^{\prime \prime} .
\end{gathered}
$$

This shows that in dimensions less than or equal to $2 \max (p, q)-1$ the restriction to the fiber is an injection of the cohomology level.

$$
i^{*} T C_{r}(\phi)=\tau i^{*} c_{r}=\tau c_{r}^{\prime} \otimes 1+1 \otimes \tau c_{r}^{\prime \prime}=i^{*} \tau c_{r}
$$

so

$$
T C_{r}(\phi)=\tau c_{r} \text { for } r \leqslant \max (p, q) .
$$

(d) $\operatorname{Sp}(p+q) / \operatorname{Sp}(p) \times \operatorname{Sp}(q), p+q>2$. Then if $P_{r}$ are the symplectic Pontryagin forms on $\operatorname{Sp}(p+q)$ we have $T P_{r}(\phi)=\tau p_{r}$ for $r \leqslant \max (p, q)$. Let $T \subset \mathrm{Sp}(p) \times \mathrm{Sp}(q) \subset \mathrm{Sp}(p+q)$ be the standard maximal torus. The generators of $H^{*}(\mathrm{Sp}(p+q))$ are $\tau p_{r}$ where $p_{r}$ may be identified with $\sigma_{r}\left(\theta_{1}^{2}, \ldots, \theta_{p+q}^{2}\right)$. The generators of $H^{*}(\operatorname{Sp}(p+q))$ are the transgressions of $p_{j}^{\prime} \times 1,1 \times p_{k}^{\prime \prime}$ which may be identified with $\sigma_{j}\left(\theta_{1}^{2}, \ldots, \theta_{p}^{2}\right) \times 1,1$ $\times \sigma_{k}\left(\theta_{p+1}^{2}, \ldots, \theta_{p+q}^{2}\right), j=1, \ldots, p$ and $k=1, \ldots, q$. Consider the commutative diagram: 


$$
\begin{aligned}
& H^{*}(\mathrm{Sp}(p) \times \mathrm{Sp}(q)) \stackrel{i^{*}}{\longleftarrow} H^{*}(\mathrm{Sp}(p+q)) \\
& \prod_{H^{*}\left(B_{\mathrm{Sp}(p) \times \mathrm{Sp}(q)}\right)} \uparrow_{i^{*}} \tau H^{*}\left(B_{\mathrm{Sp}(p+q)}\right) \\
& i^{*} \tau p_{r}=i^{*} \tau \sigma_{r}\left(\theta_{1}^{2}, \ldots, \theta_{p+q}^{2}\right)=i^{*} \frac{(-1)^{r+1}}{r} \tau S_{r}\left(\theta_{1}^{2}, \ldots, \theta_{p+q}^{2}\right) \\
& =\frac{(-1)^{r+1}}{r} \tau\left(S_{r}\left(\theta_{1}^{2}, \ldots, \theta_{p}^{2}\right) \otimes 1+1 \otimes S_{r}\left(\theta_{p+1}^{2}, \ldots, \theta_{p+q}^{2}\right)\right) \\
& =\tau \sigma_{r}\left(\theta_{1}^{2}, \ldots, \theta_{p}^{2}\right) \otimes 1+1 \otimes \tau \sigma_{r}\left(\theta_{p+1}^{2}, \ldots, \theta_{p+q}^{2}\right) \\
& =\tau p_{r}^{\prime} \otimes 1+1 \otimes \tau p_{r}^{\prime \prime} \text {. }
\end{aligned}
$$

This shows that in dimensions less than or equal to $4 \max (p, q)-1$ restriction to the fiber is an injection on the cohomology level. $i^{*} T P_{r}(\phi)=\tau i^{*} p_{r}^{\prime} \times 1$ $+1 \times \tau p_{r}^{\prime \prime}$. So $T P_{r}(\phi)=\tau p_{r}$ for $r \leqslant \max (p, q)$.

(e) $S O(2 p+2 q) / S O(2 p) \times S O(2 q), p+q>2$. Then if $P_{i}$ are the Pontryagin forms on $S O(2 p+2 q)$ we have $T P_{i}(\phi)=\tau p_{i}$ for $i \leqslant \max (p-1, q-1)$. Let $T \subset S O(2 p) \times S O(2 q) \subset S O(2 p+2 q)$ be the standard maximal torus of $S O(2 p+2 q)$. The generators of $H^{*}(S O(2 p+2 q))$ are the transgressions of the generators of $H^{*}\left(B_{S O(2 p+2 q)}\right)$ and these may be identified with $p_{r}$ $=\sigma_{r}\left(\theta_{1}^{2}, \ldots, \theta_{p+q}^{2}\right), r=1, \ldots, p+q-1$ and $x=\theta_{1}, \ldots, \theta_{p+q}$. The generators of $H^{*}(S O(2 p) \times S O(2 q))$ are the transgressions of the generators of $H^{*}\left(B_{S O}(2 p) \times S O(2 q)\right)$ and may be identified with $p_{i}^{\prime} \times 1=\sigma_{i}\left(\theta_{1}^{2}, \ldots, \theta_{p}^{2}\right) \times 1, \chi^{\prime}$ $=\theta_{1}, \ldots, \theta_{p} \otimes 1,1 \times p_{j}^{\prime \prime}=1 \times \sigma_{j}\left(\theta_{p+1}^{2}, \ldots, \theta_{p+q}^{2}\right), \chi^{\prime \prime}=1 \otimes \theta_{p+1}, \ldots, \theta_{p+q}$, $i=1, \ldots, p-1$ and $j=1, \ldots, q-1$. Consider the diagram:

$$
\begin{aligned}
& H^{*}(S O(2 p) \times S O(2 q)) \stackrel{i^{*}}{\longleftarrow} H^{*}(S O(2 p+2 q)) \\
& H^{*}\left(B_{S O(2 p) \times S O(2 q)} \stackrel{i^{*}}{\longleftarrow} H^{*}\left(B_{S O(2 p+2 q)}\right)\right. \\
& i^{*} \tau p_{r}=i^{*} \tau \sigma_{r}\left(\theta_{1}^{2}, \ldots, \theta_{p+q}^{2}\right) \\
&=i^{*} \tau(-1)^{r+1} / r S_{r}\left(\theta_{1}^{2}, \ldots, \theta_{p+q}^{2}\right) \\
&= \tau(-1)^{r+1} / r\left(S_{r}\left(\theta_{1}^{2}, \ldots, \theta_{p}^{2}\right) \times 1+1 \times S_{r}\left(\theta_{p+1}^{2}, \ldots, \theta_{p+q}^{2}\right)\right) \\
&= \tau \sigma_{r}\left(\theta_{1}^{2}, \ldots, \theta_{p}^{2}\right) \times 1+1 \times \tau \sigma_{r}\left(\theta_{p+1}^{2}, \ldots, \theta_{p+q}^{2}\right) \\
&= \tau p_{r}^{\prime} \times 1+1 \times \tau p_{r}^{\prime \prime} .
\end{aligned}
$$


So restriction to the fiber is an injection in dimensions $\leqslant 4 \max (p-1, q-1)$ -1. $i^{*} T P_{r}(\phi)=i^{*} p_{r}=\tau p_{r}^{\prime} \times 1+1 \times \tau p_{r}^{\prime \prime}$. Therefore $T P_{r}(\phi)=\tau p_{r}$ for $r$ $\leqslant \max (p-1, q-1)$.

(f) $S O(2 p+2 q+1) / S O(2 p) \times S O(2 q+1), p+q>2$. Then if $P_{i}$ are the Pontryagin forms on $S O(2 p+2 q+1), T P_{i}(\phi)=\tau p_{i}, i \leqslant \max (p-1, q)$. Let $T \subset S O(2 p) \times S O(2 q+1) \subset S O(2 p+2 q+1)$ be the standard maximal torus. The generators of $H^{*}(S O(2 p+2 q+1))$ are the transgressions of the canonical Pontryagin forms which may be identified with $P_{r}=\sigma_{r}\left(\theta_{1}^{2}, \ldots, \theta_{p+q}^{2}\right)$, $r=1, \ldots, p+q$. The generators of $H^{*}(S O(2 p) \times S O(2 q+1))$ are the transgressions of the generators of $H^{*}\left(B_{S O}(2 p) \times S O(2 q+1)\right)$ which may be identified

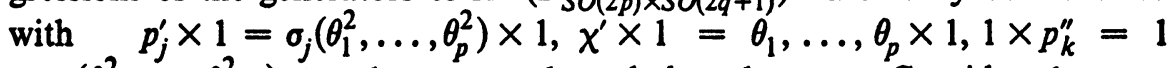
$\times \sigma_{k}\left(\theta_{1}^{2}, \ldots, \theta_{p+q}^{2}\right), j=1, \ldots, p-1$ and $k=1, \ldots, q$. Consider the commutative diagram:

$$
\begin{aligned}
& H^{*}(S O(2 p) \times S O(2 q)) \stackrel{i^{*}}{\longleftarrow} H^{*}(S O(2 p+2 q)) \\
& H^{*}\left(B_{S O(2 p) \times S O(2 q)} \stackrel{i^{*}}{\longleftarrow} H^{*}\left(B_{S O(2 p+q)}\right)\right. \\
i^{*} \tau p_{r}= & i^{*} \tau \sigma_{r}\left(\theta_{1}^{2}, \ldots, \theta_{p+q}^{2}\right) \\
= & i * \tau \frac{(-1)^{r+1}}{r} S_{r}\left(\theta_{1}^{2}, \ldots, \theta_{p+q}^{2}\right) \\
= & \tau \frac{(-1)^{r+1}}{r}\left(S_{r}\left(\theta_{1}^{2}, \ldots, \theta_{p}^{2}\right) \times 1+1 \times S_{r}\left(\theta_{p+1}^{2}, \ldots, \theta_{p+q}^{2}\right)\right) \\
= & \tau\left(\sigma_{r}\left(\theta_{1}^{2}, \ldots, \theta_{p}^{2}\right) \times 1+1 \times \sigma_{r}\left(\theta_{p+1}^{2}, \ldots, \theta_{p+q}^{2}\right)\right) \\
= & \tau p_{r}^{\prime} \times 1+1 \times \tau p_{r}^{\prime \prime} .
\end{aligned}
$$

So restriction to the fiber is an injection in dimensions $\leqslant 4 \max (p-1, q)$ -1. $i^{*} T P_{r}(\theta)=\tau i^{*} p_{r}=\tau p_{r}^{\prime} \times 1+1 \times \tau p_{r}^{\prime \prime}$. Therefore $T P_{r}(\theta)=\tau p_{r}$ for $r$ $\leqslant \max (p-1, q)$.

(g) $S O(2 p+2 q+2) / S O(2 p+1) \times S O(2 q+1), p+q>1$. Then if $P_{i}$ are the Pontryagin forms on $S O(2 p+2 q+2)$ we have $T P_{i}(\phi)=\tau p_{i}$ for $i$ $\leqslant \max (p, q)$. Let $T^{\prime} \times T^{\prime \prime} \subset T$ be standard maximal tori of $S O(2 p+1)$ $\times S O(2 q+1)$ and $S O(2 p+2 q+2)$ respectively. The generators of $H^{*}(S O(2 p+2 q+2))$ are the transgressions of the generators of $H^{*}\left(B_{S O(2 p+2 q+2)}\right)$ which may be identified with $p_{j}=\sigma_{j}\left(\theta_{1}^{2}, \ldots, \theta_{p+q+1}^{2}\right)$, $\chi=\theta_{1}, \ldots, \theta_{p+q}, j=1, \ldots, p+q$. Furthermore, the generators of 
$H^{*}(S O(2 p+1) \times S O(2 q+1))$ are the transgressions of the generators of $H^{*}\left(B_{S O}(2 p+1) \times S O(2 q+1)\right)$ which may be identified with $p_{j}^{\prime} \times 1=\sigma_{j}\left(\theta_{1}^{2}, \ldots, \theta_{p}^{2}\right)$ $\times 1,1 \times p_{k}^{\prime \prime}=1 \times \sigma_{k}\left(\theta_{p+1}^{2}, \ldots, \theta_{p+q}^{2}\right), j=1, \ldots, p$ and $k=1, \ldots, q$. Consider the commutative diagram:

$$
\begin{aligned}
& H^{*}(S O(2 p+1) \times S O(2 q+1)) \stackrel{i^{*}}{\longleftarrow} H^{*}(S O(2 p+2 q+2))
\end{aligned}
$$

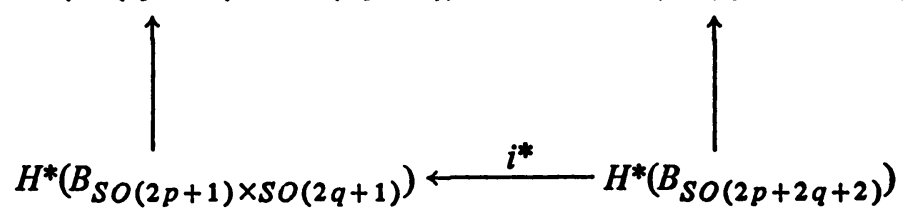

$$
\begin{aligned}
& i^{*} \tau p_{r}=i^{*} \tau \sigma_{r}\left(\theta_{1}^{2}, \ldots, \theta_{p+q+1}^{2}\right)=i^{*} \tau \frac{(-1)^{r+1}}{r} S_{r}\left(\theta_{1}^{2}, \ldots, \theta_{p+q+1}^{2}\right) \\
& =\tau \frac{(-1)^{r+1}}{r}\left(S_{r}\left(\theta_{1}^{2}, \ldots, \theta_{p}^{2}\right) \times 1+1 \times S_{r}\left(\theta_{p+1}^{2}, \ldots, \theta_{p+q}^{2}\right)\right) \\
& =\tau\left(\sigma_{r}\left(\theta_{1}^{2}, \ldots, \theta_{p}^{2}\right) \times 1+1 \times \sigma_{r}\left(\theta_{p+1}^{2}, \ldots, \theta_{p+q}^{2}\right)\right) \\
& =\tau p_{r}^{\prime} \times 1+1 \times \tau p_{r}^{\prime \prime} .
\end{aligned}
$$

So restriction to the fiber is an injection in dimensions $\leqslant 4 \max (p, q)-1$. $i^{*} T P_{r}(\theta)=\tau i^{*} p_{r}=\tau p_{r}^{\prime} \times 1+1 \times \tau p_{r}^{\prime \prime}$. Therefore $T P_{r}(\phi)=\tau p_{r}$ for $r$ $\leqslant \max (p, q)$.

(h) $S U(2 k+1) / S O(2 k+1)$. Then if $C_{j}$ denote the Chern forms on $S U(2 k+1) T C_{2 j}(\phi)$ has component $\tau c_{2 j}$ in the ring generated by the $\tau c_{2 i}$ for $j$ $\leqslant k . H^{*}(S U(2 k+1))$ is generated by the transgressions of the generators of $H^{*}\left(B_{S U(2 k+1)}\right)$ which may be identified with $c_{j}=\sigma_{j}\left(\delta_{1}, \ldots, \delta_{2 k},-\sum \delta_{i}\right), 2$ $\leqslant j \leqslant 2 k+1 . H^{*}(S O(2 k+1))$ is generated by the transgressions of the generators of $H^{*}\left(B_{S O(2 k+1)}\right)$ which may be identified with $p_{j}\left(\theta_{1}^{2}, \ldots, \theta_{k}^{2}\right), j$ $=1, \ldots, k$. If $T^{\prime} \subset T$ are maximal tori of $S O(2 k+1), S U(2 k+1)$ respectively then the restriction map on cohomology is given by $\delta_{i} \rightarrow \theta_{i}, 1 \leqslant i \leqslant k$; $\delta_{i} \rightarrow-\theta_{i}, k+1 \leqslant i \leqslant 2 k$. Now consider the diagram:

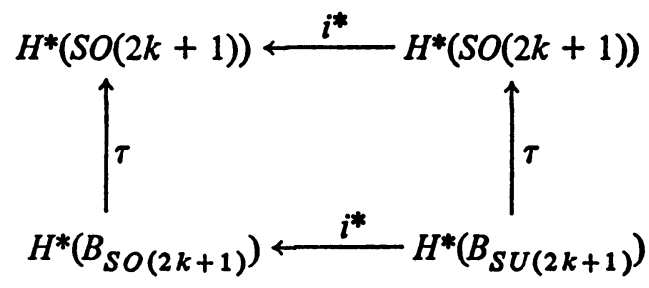




$$
\begin{aligned}
i^{*} \tau c_{2 j} & =i^{*} \tau \frac{(-1)^{2 j+1}}{2 j} S_{2 j}\left(\delta_{1}, \ldots, \delta_{2 k},-\sum \delta_{i}\right) \\
& =\tau \frac{(-1)^{2 j+1}}{j} S_{j}\left(\theta_{1}^{2}, \ldots, \theta_{k}^{2}\right) \\
& =\tau(-1)^{j} \sigma_{j}\left(\theta_{1}^{2}, \ldots, \theta_{k}^{2}\right)=(-1)^{j} \tau p_{j} .
\end{aligned}
$$

Therefore restriction to the fiber is an injection restricted to the ring generated by the $\tau c_{2 i}$ in dimensions $4 j-1$ for $j \leqslant k$.

$$
i^{*} T C_{2 j}(\phi)=\tau i^{*} c_{2 j}=(-1)^{j} \tau p_{j}
$$

Therefore $T C_{2 j}(\phi)$ has component $\tau c_{2 j}$ for $j \leqslant k$.

(i) $S U(2 k) / S O(2 k)$. Then if $C_{2 j}$ denote the canonical Chern forms of $S U(2 k), T C_{2 j}(\phi)$ has component $\tau c_{2 j}, j \leqslant k-1$, in the ring generated by the transgressions of the generators of $H^{*}\left(B_{S U(2 k)}\right)$ which may be identified with $c_{j}=\sigma_{j}\left(\delta_{1}, \ldots, \delta_{2 k-1},-\sum \delta_{i}\right), j=2, \ldots, 2 k . H^{*}(S O(2 k))$ is generated by the transgressions of the generators of $H^{*}\left(B_{S O(2 k)}\right)$ which may be identified with $p_{j}=\sigma_{j}\left(\theta_{1}^{2}, \ldots, \theta_{k}^{2}\right), j=1, \ldots, k-1$ and $\chi=\theta_{1}, \ldots, \theta_{k}$. If $T^{\prime} \subset T$ are maximal tori of $S O(2 k), S U(2 k)$ respectively then the restriction map on cohomology is given by $\delta_{i} \rightarrow \theta_{i}, i=1, \ldots, k$, and $\delta_{i} \rightarrow-\theta_{i}, i=k+1, \ldots$, $2 k-1$. Now consider the diagram:

$$
\begin{gathered}
H_{\uparrow}^{*}(S O(2 k)) \stackrel{i^{*}}{\tau} H^{*}(S U(2 k)) \\
H^{*}\left(B_{S O(2 k)}\right) \\
i^{*} \tau c_{2 j}=i^{*} \tau \frac{(-1)^{2 j+1}}{2 j} S_{2 j}\left(\delta_{1}, \ldots, \delta_{2 k-1},-\sum \delta_{i}\right) \\
=\tau(-1)^{2 j+1} / j S_{j}\left(\theta_{1}^{2}, \ldots, \theta_{k}^{2}\right)=(-1)^{j} \tau \sigma_{j}\left(\theta_{1}^{2}, \ldots, \theta_{k}^{2}\right) \\
=(-1)^{j} \tau p_{j} .
\end{gathered}
$$

Therefore restriction to the fiber is an injection restricted to the ring generated by the $\tau c_{2 i}$ in dimensions $4 j-1$ for $j \leqslant k-1 . i^{*} T C_{2 j}(\phi)=\tau i^{*} c_{2 j}$ $=(-1)^{j} \tau p_{j}$. Therefore $T C_{2 j}(\phi)$ has component $\tau c_{2 i}$ in the ring generated by the $\tau c_{2 i}$ for $j \leqslant k-1$.

(j) $S U(2 k) / \mathrm{Sp}(k), k>1$. If $C_{2 j}$ denotes the canonical Chern form on $S U(2 k) T C_{2 j}(\phi)$ has component $\tau c_{2 j}$ in the ring generated by the $\tau c_{2 i}$ for $j$ 
$\leqslant k$. The generators of $H^{*}(S U(2 k))$ are the transgressions of the generators of $H^{*}\left(B_{S U(2 k)}\right)$ which may be identified with $c_{j}=\sigma_{j}\left(\delta_{1}, \ldots, \delta_{2 k-1},-\sum \delta_{i}\right), j$ $=2, \ldots, 2 k$. The generators of $H^{*}(\operatorname{Sp}(k))$ are the transgressions of the generators of $H^{*}\left(B_{\mathrm{Sp}(k)}\right)$ and these may be identified with $p_{j}=\sigma_{j}\left(\theta_{1}^{2}, \ldots, \theta_{k}^{2}\right)$, $j=1, \ldots, k$. If $T^{\prime} \subset T$ are respectively the maximal tori of $\mathrm{Sp}(k), S U(2 k)$ then the restriction map on cohomology is given by $\delta_{i} \rightarrow \theta_{i}, i=1, \ldots, k$, and $\delta_{i} \rightarrow-\theta_{i}, i=1, \ldots, 2 k-1$. Consider the commutative diagram:

$$
\begin{gathered}
\prod_{H^{*}\left(B_{S U(2 k)}\right) \stackrel{i^{*}}{\longleftarrow} H^{*}\left(B_{\mathrm{Sp}(k)}\right)} \prod^{i^{*}} H^{*}(\operatorname{Sp}(k)) \\
i^{*} \tau c_{2 j}=i^{*} \tau \sigma_{2 j}\left(\delta_{1}, \ldots, \delta_{2 k-1},-\sum \delta_{i}\right) \\
=i^{*} \tau \frac{(-1)^{2 j+1}}{2 j} S_{2 j}\left(\delta_{1}, \ldots, \delta_{2 k-1},-\sum \delta_{i}\right) \\
=\tau \frac{(-1)^{2 j+1}}{j} S_{j}\left(\theta_{1}^{2}, \ldots, \theta_{k}^{2}\right)=\tau(-1)^{j} \sigma_{j}\left(\theta_{1}^{2}, \ldots, \theta_{k}^{2}\right) \\
=(-1)^{j} \tau p_{j} .
\end{gathered}
$$

Therefore restriction to the fiber is an injection for elements in the ring generated by the $\tau c_{2 i}$ for $i \leqslant k . i^{*} T C_{2 j}(\phi)=\tau i^{*} c_{2 j}=i^{*} \tau c_{2 j}=(-1)^{j} / 2 \tau p_{j}$. Therefore $T C_{2 j}(\phi)$ has component $\tau c_{2 j}$ in the ring generated by the $\tau c_{2 i}$.

Our results are summarized in the table below:

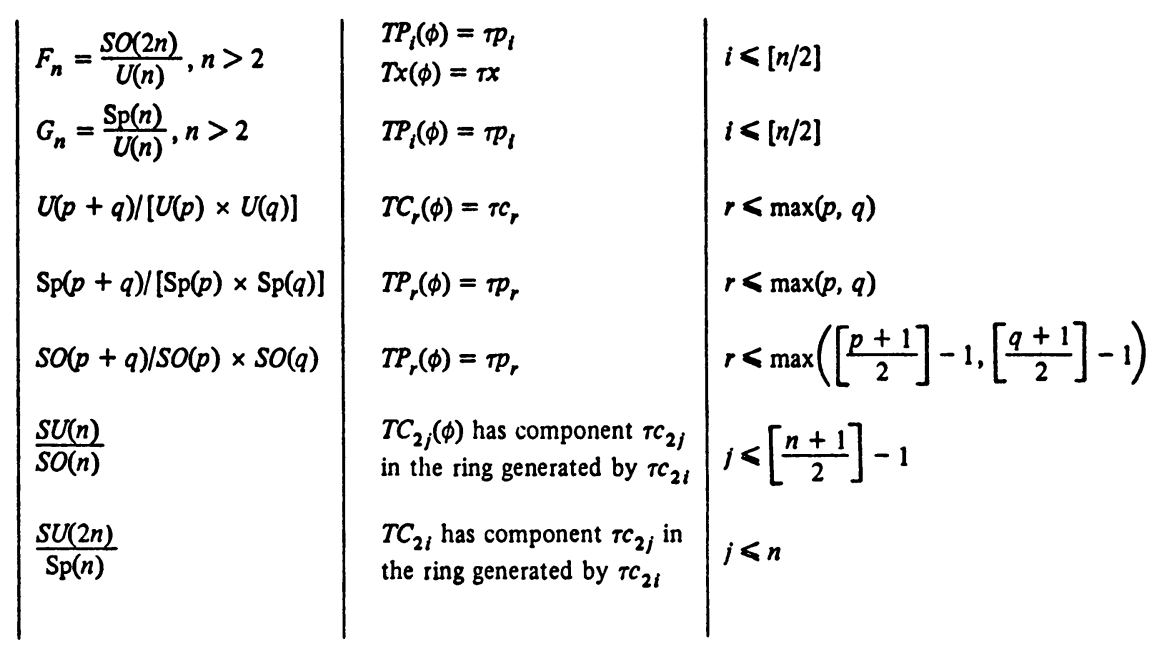


8. Chern-Simons invariants on symmetric spaces. The Chern-Simons invariants obstructing conformal immersions are by Theorems 1.1 and 4.5 associated to the polynomials $i_{s}^{*} p_{i}^{\perp}$. That is, a necessary condition that $G / H$ admit a conformal immersion in $R^{n+k}$ is that $i_{s}^{*} p_{i}^{\perp}(\Phi)=0$ and $\frac{1}{2} T\left(i_{s}^{*} p_{i}^{\perp}\right)(\phi)$ $\in H^{4_{i-1}}(G / H, Z), i>[k / 2]$, where $n=\operatorname{dim}(G / H)$. Of the spaces considered in $\S 7$ in most cases $i_{s}^{*} P_{i}^{\perp}(\Phi) \neq 0$ and this may be deduced from the fact that the associated characteristic class does not vanish [5].

The cases where necessarily $i_{s}^{*} P_{i}^{\perp}(\Phi)=0$ are considered below. In all spaces $G / H$ the elements $i_{s}^{*} P_{i}^{\perp}$ are the restrictions of elements of $I(G)$. The spaces considered below are: $S U(k) ; S U(n) / S O(n)$, with two calculations depending upon the parity of $n ; S U(2 k) / \mathrm{Sp}(k) ; S^{n}=S O(n+1) / S O(n)$. On $S U(2 k+1)$ and $S U(2 k+1) / S O(2 k+1)$ we obtain nonintegral invariants and consequently results on conformal immersions.

The calculations are based on the following result of [5]:

THEOREM 8.1. Let $\rho: G \rightarrow G l(n)$ be a representation of a compact Lie group $G$ and $\rho^{*}$ the induced map on the cohomology of classifying spaces. Identifying the elements of $H^{*}\left(B_{G}\right)$ with their images in $H^{*}\left(B_{T}\right)$ where $T$ is a maximal torus we have:

$$
\rho^{*}(p)=\underset{j}{\pi}\left(1+\omega_{j}\right)
$$

where $p$ is the Pontryagin polynomial and $\omega_{j}$ are the weights of the representation.

(a) $S U(k)$. The weights of the adjoint representation are $\theta_{i}-\theta_{j}, 1 \leqslant i \leqslant j$ $\leqslant k$, where we define $\theta_{k}=-\sum_{i=1}^{k-1} \theta_{i}$.

$$
\begin{aligned}
\tau \operatorname{Ad}^{*} p_{l}^{\perp} & =-\tau \operatorname{Ad}^{*} p_{l}=-\tau \sigma_{l}\left(\left(\theta_{i}-\theta_{j}\right)^{2}\right) \\
& =-\tau \frac{(-1)^{l+1}}{l} S_{l}\left(\left(\theta_{i}-\theta_{j}\right)^{2}\right) \\
& =\frac{(-1)^{l}}{2 l} \tau\left(\sum_{i, j} \theta_{i}^{2 l}-2 l \theta_{i}^{2 l-1} \theta_{j}+\cdots+\theta_{j}^{2 l}\right) \\
& =\frac{(-1)^{l}}{l} \tau k S_{2 l}\left(\theta_{i}\right)=\frac{(-1)^{l}}{l} 2 k l(-1)^{2 l+1} \tau \sigma_{2 l}\left(\theta_{i}\right) \\
& =2(-1)^{l+1} k \tau c_{2 l} .
\end{aligned}
$$

Let $\sigma$ be a section of the bundle of bases by left invariant vector fields. Corollary 6.4 gives $\sigma^{*} \frac{1}{2} T P_{l}^{\perp}(\theta)=\frac{1}{4} \tau \mathrm{Ad}^{*} . p_{l}^{\perp}=\frac{1}{2}(-1)^{l+1} k \tau c_{2 l}$. Since $\tau c_{2 l}$ 
generate a direct summand in $H^{*}(S U(k), Z)$ [4] the Chern-Simons invariants do not vanish for $k$ odd.

THEOREM 8.2. $S U(2 k+1)$ with bi-invariant metric does not conformally immerse in Euclidean space of codimension $2 k-1$.

Proof. Apply Theorems 1.1, 4.5 and the calculation above.

(b) $S U(2 k) / S O(2 k)$. Every $S O(2 k)$ invariant polynomial in the ring generated by the Pontryagin polynomials is the restriction of an $S U(2 k)$ invariant polynomial. The weights of the isotropy representation are $2 \theta_{i}, \theta_{i}-\theta_{j}, \theta_{i}+\theta_{j}$, $1 \leqslant i \leqslant j \leqslant k . \quad i_{s}^{*} p_{l}=\sigma_{l}\left(4 \theta_{i}^{2},\left(\theta_{i}-\theta_{j}\right)^{2},\left(\theta_{i}+\theta_{j}\right)^{2}\right)$. This is seen to lie in the ring generated by the Pontryagin forms by an easy induction argument using the formula of (7) relating the $S_{i}$ and $\sigma_{i}$.

$$
\begin{aligned}
\tau i_{s}^{*} p_{l}^{\perp}= & -\tau i_{s}^{*} p_{l}=-\tau \sigma_{l}\left(4 \theta_{i}^{2},\left(\theta_{i}-\theta_{j}\right)^{2},\left(\theta_{i}+\theta_{j}\right)^{2}\right) \\
= & -\tau \frac{(-1)^{l+1}}{l} S_{l}\left(4 \theta_{1}^{2},\left(\theta_{i}-\theta_{j}\right)^{2},\left(\theta_{i}+\theta_{j}\right)^{2}\right) \\
= & \frac{(-1)^{l}}{l} \tau\left(\frac{1}{2} \sum 2^{2 l} \theta_{i}^{2 l}+\frac{1}{2} \sum\left(\theta_{i}-\theta_{j}\right)^{2 l}+\left(\theta_{i}+\theta_{j}\right)^{2 l}\right) \\
= & -\tau\left(2^{2 l-1} \sigma_{l}\left(\theta_{i}^{2}\right)\right) \\
& -\frac{1}{2} \frac{(-1)^{l+1}}{l} \tau\left(\sum \theta_{i}^{2 l}-2 l \theta_{i}^{2 l-1} \theta_{j}+\cdots\right. \\
= & \left.-\theta_{j}^{2 l}+\theta_{i}^{2 l}+2 l \theta_{i}^{2 l-1} \theta_{j}+\cdots+\theta_{j}^{2 l}\right) \\
= & -2^{2 l-1} \tau \sigma_{l}\left(\theta_{i}^{2}\right)-\frac{(-1)^{l+1}}{l}\left(\sum \theta_{i}^{2 l}+\left(\begin{array}{c}
2 l \\
2
\end{array}\right) \theta_{i}^{2 l-2} \theta_{j}^{2}+\cdots+\theta_{j}^{2 l}\right) \\
= & -2^{2 l-1} \tau p_{l}-2 k \tau p_{l} \\
= & -\left(2^{2 l-1}+2 k\right) \tau p_{l} .
\end{aligned}
$$

Now $\tau c_{2 l}$ restricts to $(-1)^{l} \tau p_{l}$. Thus the component of $\frac{1}{2} T i_{s}^{*} p_{l}^{\perp}(\theta)$ in the $\tau c_{2 l}$ summand is $(-1)^{l+1}\left(2^{2 l-2}+k\right) \tau c_{2 l}$. This is always integral.

(c) $S U(2 k+1) / S O(2 k+1)$. Every $S O(2 k+1)$ invariant polynomial is the restriction of an $S U(2 k+1)$ polynomial. Therefore the Chern-Simons invariants are well defined. The weights of the isotropy representation are $\theta_{i}-\theta_{j}$, $\theta_{i}+\theta_{j}, \theta_{i}, 2 \theta_{i}, 1 \leqslant i<j \leqslant k$. 
CHERN-SIMONS INVARIANTS

163

$$
\begin{aligned}
\tau i_{s}^{*} p_{l} & =-\tau i_{s}^{*} p_{l}^{\perp}=-\tau \sigma_{l}\left(4 \theta_{i}^{2}, \theta_{i}^{2},\left(\theta_{i}+\theta_{j}\right)^{2},\left(\theta_{i}-\theta_{j}\right)^{2}\right) \\
& =\frac{(-1)^{l}}{l} \tau S_{l}\left(4 \theta_{i}^{2}, \theta_{i}^{2},\left(\theta_{i}+\theta_{j}\right)^{2},\left(\theta_{i}-\theta_{j}\right)^{2}\right) \\
& =\frac{(-1)^{l}}{l} \tau\left(\sum \theta_{i}^{2 l}+\frac{1}{2} \Sigma\left(\left(\theta_{i}+\theta_{j}\right)^{2 l}+\left(\theta_{i}-\theta_{j}\right)^{2 l}\right)+\frac{1}{2} \sum\left(2 \theta_{i}\right)^{2 l}\right) \\
& =\frac{(-1)^{l}}{l} \tau\left(\left(1+2^{2 l-1}\right) S_{l}\left(\theta_{i}^{2}\right)+\Sigma\left(\theta_{i}^{2 l}+\left(\begin{array}{c}
2 l \\
2
\end{array}\right) \theta_{i}^{2 l-2} \theta_{j}^{2}+\cdots+\theta_{j}^{2 l}\right)\right) \\
& =-\tau \sigma_{l}\left(\theta_{i}^{2}\right)\left(1+2^{2 l-1}\right)+\frac{(-1)^{l}}{l} 2 k \tau S_{l}\left(\theta_{i}^{2}\right) \\
& =-\left(1+2^{2 l-1}\right) \tau p_{l}-2 k \tau p_{l} \\
& =-\left(+2 k+1+2^{2 l-1}\right) \tau p_{l} .
\end{aligned}
$$

Now the $\tau c_{2 l}$ restrict to $(-1)^{l} \tau p_{l}$ on the fiber. Thus the component of $\frac{1}{2} T\left(i_{s}^{*} P_{l}^{\perp}\right)(\phi)$ in the $\tau c_{2 l}$ direction is $\frac{1}{2}(-1)^{l+1}\left(2 k+1+2^{2 l-1}\right) \tau c_{2 l}$. Since $\tau c_{2 l}$ generates a direct summand in $H^{*}(S U(2 k+1), Z)$ [4] this is not integral.

THEOREM 8.3. $S U(2 k+1) / S O(2 k+1)$ with $S U(2 k+1)$ invariant metric does not conformally immerse in codimension $2 k-1$.

Proof. Apply calculation above and Theorems 1.1, 4.5.

(d) $S U(2 k) / \mathrm{Sp}(k), k>1$. Every $\mathrm{Sp}(k)$ invariant polynomial is the restricion of an $S U(2 k)$ invariant polynomial. Thus the Chern-Simons invariant are defined. The weights of the isotropy representation are $\theta_{i}-\theta_{j}, \theta_{i}+\theta_{j}, 1 \leqslant i$ $<j \leqslant k$.

$$
\begin{aligned}
\tau i_{s}^{*} p_{l}^{\perp} & =-\tau i_{s}^{*} p_{l}=-\tau i_{s}^{*} p_{l}=-\tau \sigma_{l}\left(\left(\theta_{i}-\theta_{j}\right)^{2},\left(\theta_{i}+\theta_{j}\right)^{2}\right) \\
& =\frac{(-1)^{l}}{l} S_{l}\left(\left(\theta_{i}-\theta_{j}\right)^{2},\left(\theta_{i}+\theta_{j}\right)^{2}\right) \\
& =\tau \frac{(-1)^{l}}{l}\left(\frac{1}{2} \sum\left(\left(\theta_{i}-\theta_{j}\right)^{2 l}+\left(\theta_{i}+\theta_{j}\right)^{2 l}\right)-\frac{1}{2} \sum\left(2 \theta_{i}\right)^{2 l}\right) \\
& =\tau \frac{(-1)^{l}}{l}\left(\sum \theta_{i}^{2 l}+\left(\begin{array}{c}
2 l \\
2
\end{array}\right) \theta_{i}^{2 l-2} \theta_{j}^{2}+\cdots+\theta_{j}^{2 l}-2^{2 l-1} \sum \theta_{i}^{2 l}\right) \\
& =\frac{(-1)^{l+1}}{l} 2^{2 l-1} \tau S_{l}\left(\theta_{i}^{2}\right)+\tau \frac{(-1)^{l}}{l}(2 k) S_{l}\left(\theta_{i}^{2}\right) \\
& =\left(-2 k+2^{2 l-1}\right) \tau \sigma_{l}\left(\theta_{i}^{2}\right)=\left(-2 k+2^{2 l-1}\right) \tau p_{l} .
\end{aligned}
$$


Now $\tau c_{2 l}$ restricts to $(-1)^{l} \tau p_{l}$ on the fiber. Thus the component of $\frac{1}{2} T\left(i_{s}^{*} P_{l}^{\perp}\right)$ $\cdot(\phi)$ in the $\tau c_{2 l}$ direction is $(-1)^{l}\left(-k+2^{2 l-2}\right) c_{2 l}$. This is always integral.

(e) $S_{n}=S O(n+1) / S O(n), n \geqslant 3$. The isotropy representation $i_{s}^{*}: S O(n)$ $\rightarrow G l(n)$ is the canonical injection. Thus $i_{s}^{*} p_{l}^{\perp}=p_{l}^{\perp}, \frac{1}{2} T p_{l}^{\perp}(\phi)=-\frac{1}{2} \tau p_{l}$. By [6] this is a generator for the integral cohomology of $S O(n+1)$. This is to be expected since $S^{n}$ isometrically embeds in $R^{n+1}$.

\section{REFERENCES}

1. A. Adler, Characteristic classes of homogeneous spaces, Trans. Amer. Math. Soc. 86 (1957), 348-365, MR 19, 1181.

2. M. F. Atiyah, V. K. Patodi and I. M. Singer, Spectral asymmetry and Riemannian geometry. I, Proc. Cambridge Philos. Soc. 77 (1975), 43-69.

3. R. Bishop and J. Crittenden, Geometry of manifolds, Pure and Appl. Math., vol. 15, Academic Press, New York, 1964. MR 29 \#6401.

4. A. Borel, Sur la cohomologie des espaces fibrés principaux et de espaces homogènes de groupes de Lie compacts, Ann. of Math. (2) 57 (1953), 115-207. MR 14, 490.

5. A. Borel and F. Hirzebruch, Characteristic classes and homogeneous spaces. I, Amer. J. Math. 80 (1958), 458-538. MT 21 \# 1586.

6. Characteristic classes and homogeneous spaces. II, Amer. J. Math. 81 (1959), 315-382. MR 22 \#988.

7. N. Bourbaki, Eléments de mathématique. XXVI. Groupes et algèbres de Lie. Chap. I: Algèbres de Lie, Acutalités Sci. Indust., no. 1285, Hermann, Paris, 1960. MR 24 \# A2641.

8. Jeff Cheeger and James Simons, Differential characters and geometric invariants (preprint).

9. S. S. Chern, Geometry of characteristic classes, Proc. Thirteenth Biennial Seminar Canadian Math. Soc., 1972, pp. 1-40.

10. On the characteristic classes of complex sphere bundles and algebraic varieties, Amer.

J. Math. 75 (1953), 565-597. MR 15, 154.

11. S. S. Chern and J. Simons, Characteristic forms and geometric invariants, Ann. of Math. 99 (1974), 48-69.

12. J. L. Heitsch and H. B. Lawson, Transgressions, Chern-Simons invariants and the classical groups, J. Differential Geometry 9 (1974), 423-434.

13. S. Kobayashi and K. Nomizu, Foundations of differential geometry. Vol. I, Interscience, New York, 1963. MR 27 \#2945.

14. - Foundations of differential geometry. Vol. II, Interscience Tracts in Pure and Appl. Math., no. 15, vol. II, Interscience, New York, 1969. MR 38 \#6501.

15. J. L. Koszul, Homologie et cohomologie des algèbres de Lie, Bull. Soc. Math. France 78 (1950), 65-127. MR 12, 120.

16. R. Narishimhan and S. Ramanan, Existence of universal connections. II, Amer. J. Math. 85 (1963), 223-231.

17. K. Nomizu, Invariant affine connections on homogeneous spaces, Amer. J. Math. 76 (1954), 33-65. MR 15, 468.

18. J. Simons, Characteristic forms and transgression. II: Characters associated to a connection (preprint).

19. B. L. van der Waerden, Modern algebra. Vol. I, Springer, Berlin, 1931; English transl., Ungar, New York, 1949. MR 10, 587.

20. J. Wolf, Spaces of constant curvature, Dept. of Math., University of California, Berkeley, Calif., 1972.

Department of Mathematics, Massachusetts institute of Technology, Cambridge, MASSACHUSETTS 02139

Current address: Department of Mathematics, The John Hopkins University, Baltimore, Maryland 21218 